\title{
Autophagy is defective in collagen VI muscular dystrophies, and its reactivation rescues myofiber degeneration
}

\author{
Paolo Grumati $^{1,8}$, Luisa Coletto ${ }^{2,8}$, Patrizia Sabatelli ${ }^{3}$, Matilde Cescon ${ }^{1}$, Alessia Angelin ${ }^{4}$, Enrico Bertaggia ${ }^{2}$, \\ Bert Blaauw $^{5}$, Anna Urciuolo ${ }^{1}$, Tania Tiepolo ${ }^{1}$, Luciano Merlini ${ }^{6}$, Nadir M Maraldi ${ }^{3,7}$, Paolo Bernardi ${ }^{4}$, \\ Marco Sandri ${ }^{2,4} \&$ Paolo Bonaldo ${ }^{1}$
}

\begin{abstract}
Autophagy is crucial in the turnover of cell components, and clearance of damaged organelles by the autophagic-lysosomal pathway is essential for tissue homeostasis. Defects of this degradative system have a role in various diseases, but little is known about autophagy in muscular dystrophies. We have previously found that muscular dystrophies linked to collagen VI deficiency show dysfunctional mitochondria and spontaneous apoptosis, leading to myofiber degeneration. Here we demonstrate that this persistence of abnormal organelles and apoptosis are caused by defective autophagy. Skeletal muscles of collagen VI-knockout (Col6a $1^{-/}$) mice had impaired autophagic flux, which matched the lower induction of beclin-1 and BCL-2/adenovirus E1Binteracting protein-3 (Bnip3) and the lack of autophagosomes after starvation. Forced activation of autophagy by genetic, dietary and pharmacological approaches restored myofiber survival and ameliorated the dystrophic phenotype of $\mathrm{Col}_{6 \mathrm{a} 1^{-/-}}$mice. Furthermore, muscle biopsies from subjects with Bethlem myopathy or Ullrich congenital muscular dystrophy had reduced protein amounts of beclin-1 and Bnip3. These findings indicate that defective activation of the autophagic machinery is pathogenic in some congenital muscular dystrophies.
\end{abstract}

Macroautophagy (hereafter referred to as autophagy) is a dynamic process in which portions of cytoplasm are sequestered within double-membraned vesicles called autophagosomes and delivered to lysosomes for degradation and subsequent recycling ${ }^{1-3}$. The autophagic machinery is highly conserved and has key roles in tissue homeostasis, participating in the clearance of damaged organelles, misfolded proteins and pathogens ${ }^{1-4}$. Furthermore, autophagy is crucial for cell survival during nutrient deprivation, but it is detrimental when massively activated or inhibited ${ }^{2}$. Likewise, mitochondria are essential for energy conservation, but, if damaged, they become a source of proapoptotic factors and reactive oxygen species $^{5,6}$. Selective removal of dysfunctional mitochondria via autophagy (mitophagy) is a key mechanism to not only preserve cell viability but also rejuvenate mitochondrial function? ${ }^{7}$.

Congenital muscular dystrophies represent a large and heterogeneous group of inherited muscle disorders with a severe and progressive clinical course. Mutations in any of the three genes coding for collagen VI, a major extracellular matrix protein of the endomysium of skeletal muscles, cause multiple muscle diseases, including Bethlem myopathy and Ullrich congenital muscular dystrophy (UCMD) ${ }^{8}$. We have previously demonstrated that muscles of Col6a1 ${ }^{-/-}$mice and humans with UCMD or Bethlem myopathy have a latent mitochondrial dysfunction accompanied by ultrastructural alterations of mitochondria and the sarcoplasmic reticulum and spontaneous apoptosis of muscle fibers $^{9,10}$ (see also Supplementary Fig. 1a). However, in our previous study we did not uncover the reason for the occurrence of dysfunctional organelles. Here we show that the accumulation of abnormal mitochondria and sarcoplasmic reticulum is caused by a defect of autophagy and that restoration of a proper autophagic flux in $\mathrm{Col}_{6 \mathrm{a} 1^{-/-}}$muscles ameliorates these alterations.

\section{RESULTS}

Autophagy is impaired in Col6a1 $1^{-/-}$muscles

To explore the relationship between organelle defects and muscle pathology of collagen VI muscular dystrophies in vivo, we examined several skeletal muscle types of Col6a1 $1^{-/-}$mice for the main molecular pathways linked with mitochondrial function and cell survival. We chose the diaphragm and tibialis anterior as examples of oxidative and glycolytic muscles, respectively. We did not find any substantial difference between wild-type and Col6a1 $1^{-/-}$muscles in the protein amounts of B cell leukemia/lymphoma-2 (Bcl-2), Bcl-2-associated $\mathrm{X}$ protein $(\mathrm{Bax})$ and $\mathrm{Bcl}-\mathrm{X}_{\mathrm{L}}$ and in the phosphorylation of Akt, suggesting no major alterations in pro- or antiapoptotic pathways. However, the amount of the active form of AMP-activated protein kinase (AMPK) was increased in Col6a1 $1^{-1-}$ muscles (Supplementary Fig. 1b,c). AMPK acts as an energy sensor, being activated under

\footnotetext{
${ }^{1}$ Department of Histology, Microbiology \& Medical Biotechnology, University of Padova, Padova, Italy. ${ }^{2}$ Dulbecco Telethon Institute, Venetian Institute of Molecular Medicine, Padova, Italy. ${ }^{3}$ Institute of Medical Genetics, National Research Council, c/o Rizzoli Orthopaedic Institute, Bologna, Italy. ${ }^{4}$ Department of Biomedical Sciences, University of Padova, Padova, Italy. ${ }^{5}$ Department of Human Anatomy \& Physiology, University of Padova, Padova, Italy. ${ }^{6}$ Department of Experimental and Diagnostic Medicine, University of Ferrara, Ferrara, Italy. ${ }^{7}$ Department of Anatomical Sciences, University of Bologna, Bologna, Italy. ${ }^{8}$ These authors contributed equally to this work. Correspondence should be addressed to P. Bonaldo (bonaldo@bio.unipd.it) or M.S. (marco.sandri@unipd.it).
}

Received 2 June; accepted 24 September; published online 31 October 2010; corrected after print 2 March 2011; doi:10.1038/nm.2247 
metabolic stress ${ }^{11}$. These data indicate an energy imbalance in Col6 $a 1^{-/-}$muscles, which is readily explained by the observed mitochondrial dysfunction. Indeed, it was previously shown that Col6a1 ${ }^{-/-}$ flexor digitorum brevis (FDB) myofibers have an increased incidence of dysfunctional mitochondria ${ }^{9}$. The presence of abnormal mitochondria with disorganized cristae (Supplementary Fig. 1d) prompted us to check whether the autophagic system was affected. Compared to wild-type mice, muscles from Col6a1 $1^{-1-}$ mice had less of the lipidated form of microtubule-associated protein-1 light chain 3 (LC3)-II (Fig. 1a and Supplementary Fig. 1e), which is generated during autophagosome formation ${ }^{1,2}$. The decreased conversion of LC3-I to LC3-II suggested an alteration of basal autophagy in Col6a1 $1^{-1-}$ muscles and led us to investigate the autophagic process.

We next subjected mice to starvation for $24 \mathrm{~h}$, a well-characterized stimulus able to induce the formation of autophagosomes in various organs including muscles ${ }^{12}$. Fasting for $24 \mathrm{~h}$ prompted massive autophagosome formation in wild-type but not Col6a1 $1^{-/-}$muscles, as revealed by the appearance of LC3-positive puncta in tibialis anterior transfected with YFP-LC3 (Supplementary Fig. 1h) and by electron microscopy of diaphragm (Fig. 1b,c). Notably, the few autophagosomes present in Col6a1 ${ }^{-/-}$diaphragm contained mitochondria with abnormal cristae (Supplementary Fig. 1i). Moreover, diaphragm and tibialis anterior showed reduced LC3-I to LC3-II conversion in fasted Col6a1 $1^{-/-}$mice compared to fasted wild-type mice (Fig. 1d and Supplementary Fig. 1j). Tibialis anterior was completely resistant to autophagy induction, whereas diaphragm showed a partial LC3 conversion after 24-h starvation (Fig. 1d). The differential response of these two muscles probably reflects differences in metabolic properties and fiber types ${ }^{12}$. These results obtained with fasted mice confirm that Col6a1 $1^{-1-}$ muscles have an abnormal response to a physiological autophagic stimulus.

The decreased amount of autophagosomes and the reduced LC3 lipidation in Col6a1 $1^{-/-}$muscles could be caused by either defective autophagy induction or excessive vesicle exhaustion. Because autophagy is a dynamically regulated process, we used a combination of in vivo tools for reliably monitoring the autophagic flux ${ }^{13,14}$. First, we used a genetic approach to knock down mRNA encoding LC3 (Map1lc3b) and thus inhibit autophagosome formation. To achieve this knockdown in vivo, we transfected adult myofibers with a bicistronic vector encoding Map1lc3b-targeting shRNA and $\mathrm{GFP}^{15}$. Knockdown of Map1lc3b led to a significant increase in the number of TUNEL-positive nuclei in wild-type myofibers, but it did not affect the TUNEL-positive nuclei of Col6a1 $1^{-1-}$ myofibers (Fig. 1e). Next, we treated mice with chloroquine ${ }^{16}$, a lysosomal
Figure 1 Autophagy is impaired in Col6a1-/mice. (a) Western blot for LC3 lipidation in diaphragm of fed wild-type and Col6a ${ }^{-/-}$mice. MW, molecular weight; WT, wild-type; GAPDH, glyceraldehyde 3-phosphate dehydrogenase. (b) Electron-microscopic quantification of myofibers containing autophagic vesicles in diaphragms of fed and 24-h-fasted mice (*** $P<0.001 ; n=5$, each group). Error bars indicate s.d. (c) Electron micrograph of a double-membrane autophagosome (arrowheads) containing mitochondria (mit) and sarcoplasmic reticulum (sr) in diaphragms of 24-h-fasted wild-type mice. Scale bar, $400 \mathrm{~nm}$. (d) Western blot for LC3 lipidation in diaphragm (left) and tibialis anterior (right) of fed and 24-h-fasted mice. (e) Quantification of TUNEL-positive nuclei in tibialis anterior transfected with vector expressing either scrambled or Map 1/c3b-targeting shRNA ( ${ }^{*} P<$ 0.05; NS, not significant; $n=5$, each group). Error bars indicate s.e.m. (f) Western blot for LC3 lipidation in diaphragm (left) and tibialis anterior (right) of untreated mice (-) or mice treated with chloroquine diphosphate at $50 \mathrm{mg}$ per kg body weight per day for $10 \mathrm{~d}(+)$. (g) Western blot for LC3 and p62 in diaphragm (left) and tibialis anterior (right) of fed, 6-h-fasted and 12-h-fasted mice. (h) Western blot for beclin-1 and Bnip3 in diaphragm (left) and tibialis anterior (right) of fed and 24-h-fasted mice (top) and densitometric quantification of beclin-1 (middle) and Bnip3 (bottom) $\left({ }^{* * *} P<0.001\right.$; ${ }^{*} P<0.05 ; n=3$ ). Error bars indicate s.e.m. (i) Quantitative RT-PCR (qRT-PCR) analysis of Bnip3 mRNA in tibialis anterior of fed and 24-h-fasted mice $\left({ }^{*} P<0.05 ; n=7\right)$. Error bars indicate s.e.m. (j) Western blot for Akt and 4E-BP1 phosphorylation ( $p-A k t$ and $p-4 E-B P 1$, respectively) in diaphragm (left) and tibialis anterior (right) of fed or 24-h-fasted mice. a
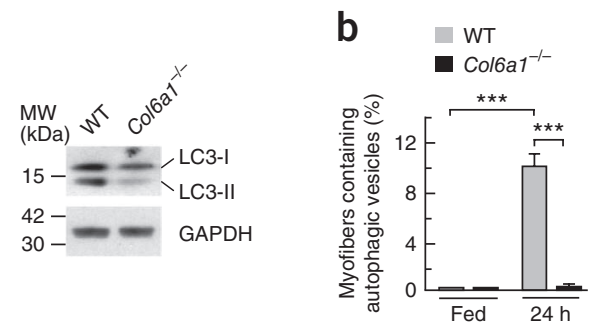

d

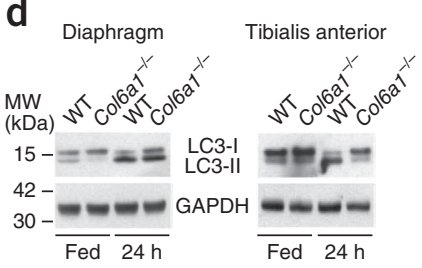

e Scramble

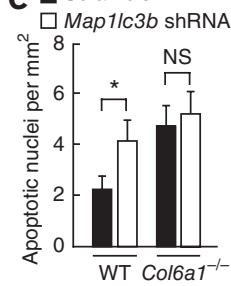

g

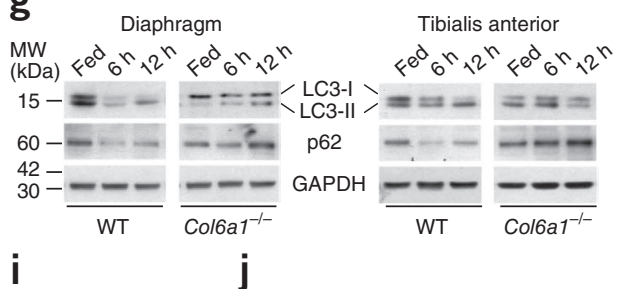

i

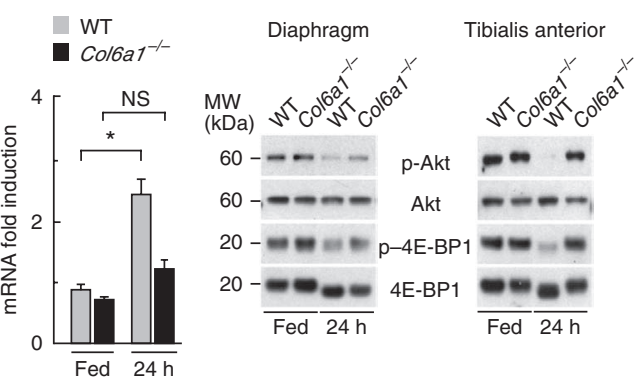

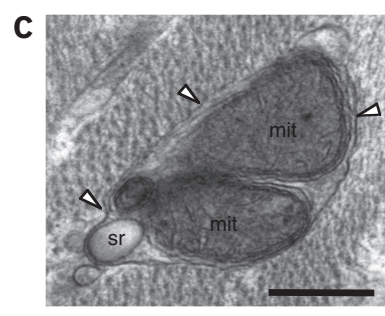

f

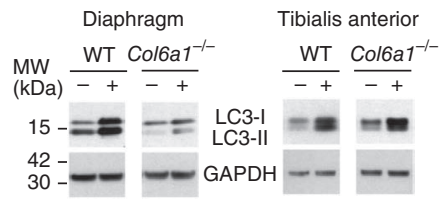

h Diaphragm Tibialis anterior
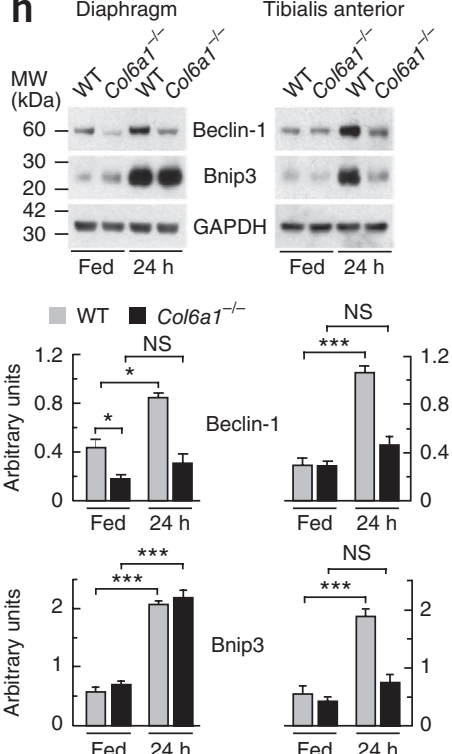
inhibitor that blocks the degradation of autophagosome content, including LC3 (refs. 13,17), and investigated its effect on LC3 protein abundance. Chloroquine treatment led to a marked increase in LC3 bands in both diaphragm and tibialis anterior of wild-type mice, but the increase was much smaller in the corresponding samples of Col6a1-/- mice (Fig. 1f and Supplementary Fig. 2a). Finally, we assessed during fasting the variations in protein amounts of p62, a well-known substrate of the autophagy-lysosome system $^{13,14,18}$. Both diaphragm and tibialis anterior showed a decrease in p62 during the first 6-12 h of fasting in wild-type mice but not in Col6a1 $1^{-/-}$mice (Fig. 1g and Supplementary Fig. 2b). Together, these data show an impairment of autophagy induction in Col6a1 $1^{-/-}$muscles, which explains their lower incidence of autophagosomes and defective LC3 lipidation.

\section{Beclin-1 and Bnip3 are defective in Col6a $1^{-/-}$muscles}

Bnip3 has a key role in the autophagic removal of mitochondria ${ }^{19,20}$, and induction of Bnip3 is crucial for autophagosome formation in muscle during starvation ${ }^{15}$. In contrast to the case in wild-type mice, Bnip3 protein and mRNA expression was not induced in tibialis anterior of 24-h-fasted Col6a1 $1^{-/-}$mice (Fig. 1h,i). Conversely, diaphragm of 24-h-fasted Col6a1 $1^{-/-}$mice showed Bnip3 induction, which matched the partial LC3 lipidation reported above (Fig. 1h). Other Bcl-2 family members, including Bax and Bcl- $\mathrm{X}_{\mathrm{L}}$, did not differ in expression between fed and fasted mice as well as between control and knockout mice, whereas Bcl-2 protein abundance was increased only in fasted Col6a1 $1^{-1-}$ tibialis anterior (Supplementary Fig. 2e). Bcl-2 has been shown to inhibit the autophagic function of beclin-1, a component of the class III phosphoinositide 3-kinase (PI3K) complex necessary for autophagosome formation ${ }^{2,21}$. Because beclin-1 plays a key part in autophagy ${ }^{2}$, we investigated its expression in wild-type and knockout muscles. Under fed conditions, beclin-1 protein amounts were lower in Col6a1-/- diaphragm when compared to wild-type diaphragm (Fig. 1h). Starvation led to a marked increase of beclin-1 protein levels in both diaphragm and tibialis anterior of wild-type animals (Fig. 1h). Notably, the levels of mRNA encoding for beclin-1 (Becn1) were similar in fed and starved tibialis anterior (Supplementary Fig. 2f), suggesting that variations of beclin-1 levels in muscle may primarily rely on protein stability. Conversely, Col6a1 $1^{-/-}$diaphragm and tibialis anterior did not show any substantial increase of beclin-1 protein after 24-h fasting (Fig. 1h). Vps34 protein, the class III PI3K of the PI3K-beclin-1 complex ${ }^{1-3}$, was upregulated in Col6a1 $1^{-1-}$ diaphragm but not in Col6a1 ${ }^{-/-}$tibialis anterior after 24-h fasting (Supplementary Fig. 2g,h). These data indicate that Col6a1 $1^{-/-}$muscles have an imbalance of proteins actively involved in the autophagic process and suggest that complete activation of autophagy requires a proper induction of beclin-1 and Bnip3.
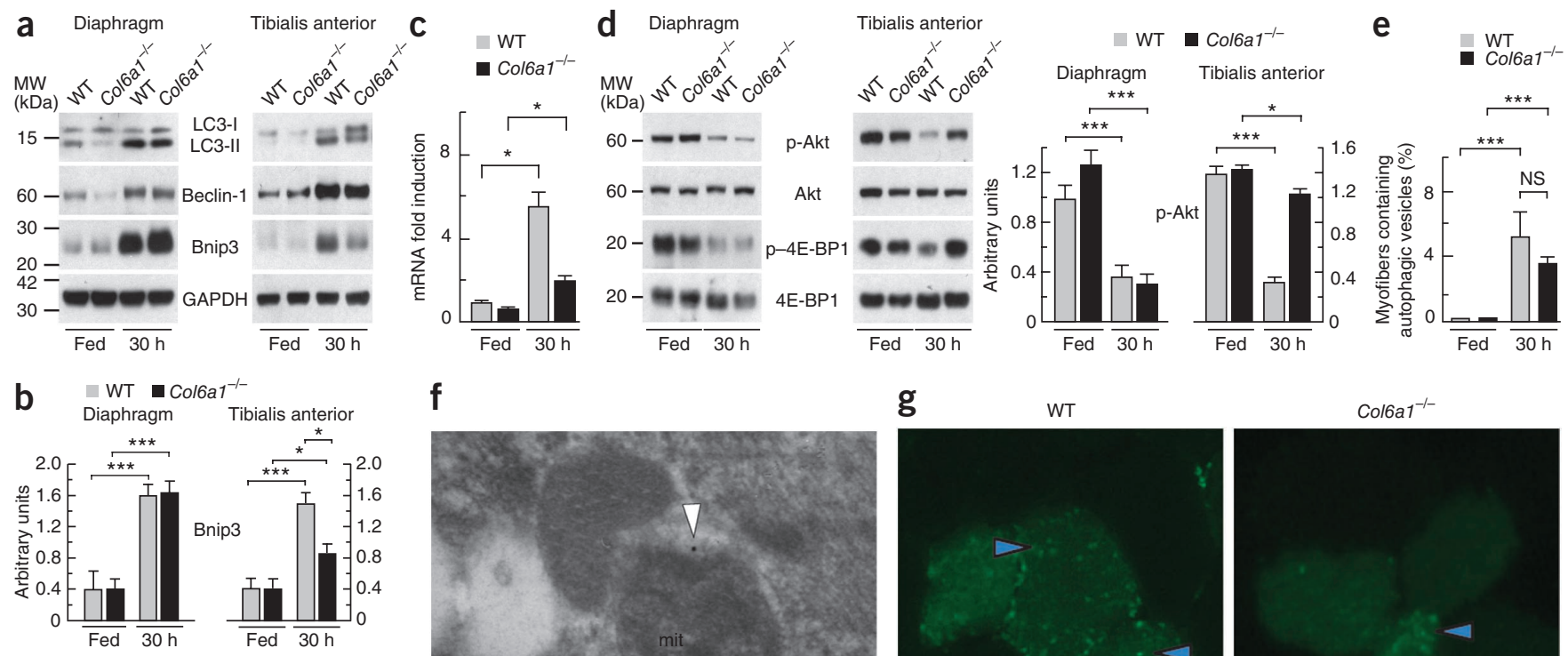

f

g

WT

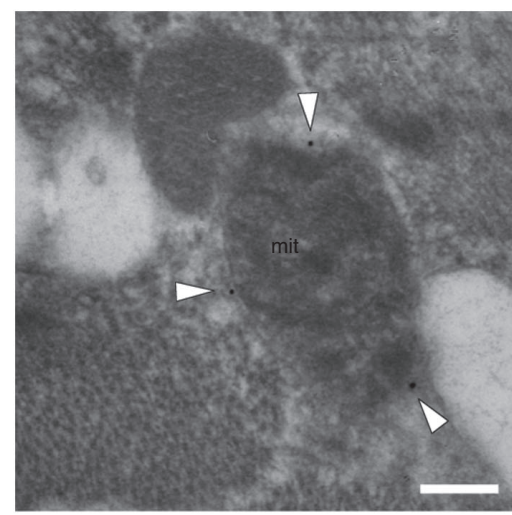

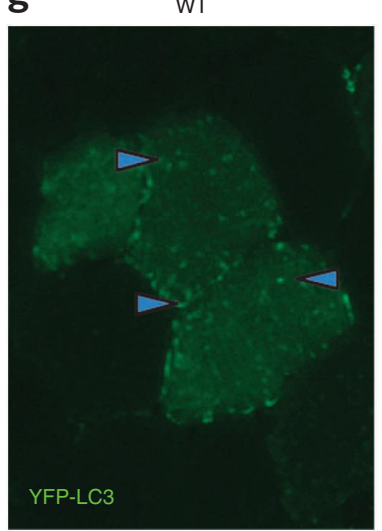

Col6a $1^{-1-}$

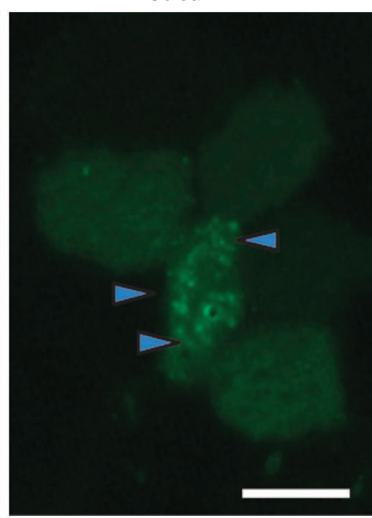

Figure 2 Prolonged starvation induces autophagy in Col6a $1^{-/}$mice. (a) Western blot for LC3, beclin-1 and Bnip3 in diaphragm (left) and tibialis anterior (right) of fed and 30-h-fasted mice. (b) Densitometric quantification of Bnip3 (top) and beclin-1 (bottom) after western blotting of diaphragm (left) and tibialis anterior (right) ( ${ }^{* *} P<0.001 ;{ }^{*} P<0.05, n=3$ ). Error bars indicate s.e.m. (c) qRT-PCR analysis of Bnip3 mRNA in tibialis anterior of fed and 30-h-fasted mice ( $\left.{ }^{*}<<0.05 ; n=7\right)$. Error bars indicate s.e.m. (d) Immunoblot analysis for Akt and 4E-BP1 phosphorylation in diaphragm (left) and tibialis anterior (right) of fed and 30-h-fasted mice. The right graphs show the densitometric quantification of the western blots for p-Akt. ( $\left.{ }^{* *} P<0.001 ;{ }^{*} P<0.05 ; n=3\right)$. Error bars indicate s.e.m. (e) Quantification of myofibers containing autophagic vesicles in diaphragms of 30-h-fasted mice (***P<0.001;n=5, each group). Error bars indicate s.d. (f) Immunogold labeling of LC3 in 30-h-fasted Col6a $1^{-/-}$diaphragm. LC3 labeling (black dots marked by arrowheads) is associated with the membrane of an autophagosome containing a mitochondrion (mit). Scale bar, $300 \mathrm{~nm}$. (g) Fluorescence microscopy of tibialis anterior cryosections from wild-type and Col6a $1^{-1-}$ mice transfected with YFP-LC3 and starved for 30 h. LC3 puncta (arrowheads) are indicated. Scale bar, $50 \mu \mathrm{m}$. 
a

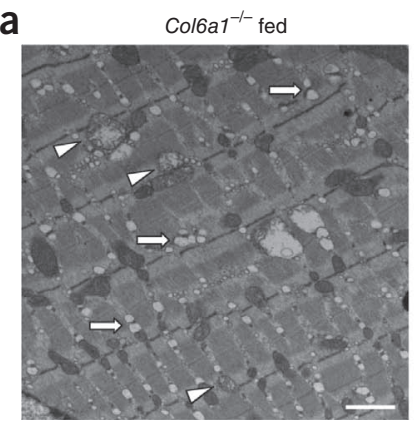

b

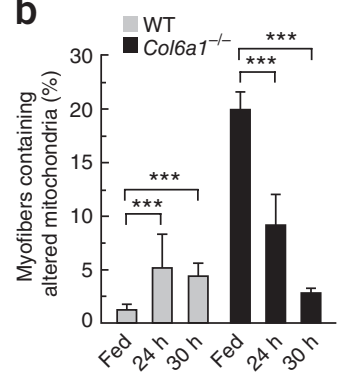

${ }_{180}$

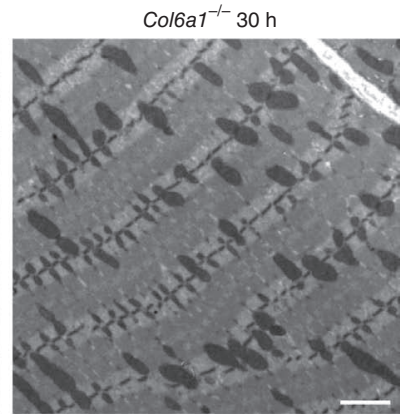

WT $24 \mathrm{~h}$

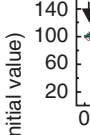

$T$ fed
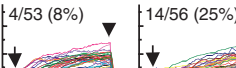

$\downarrow$

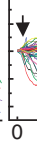

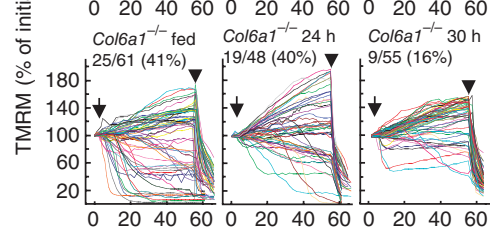

Time (min)

d
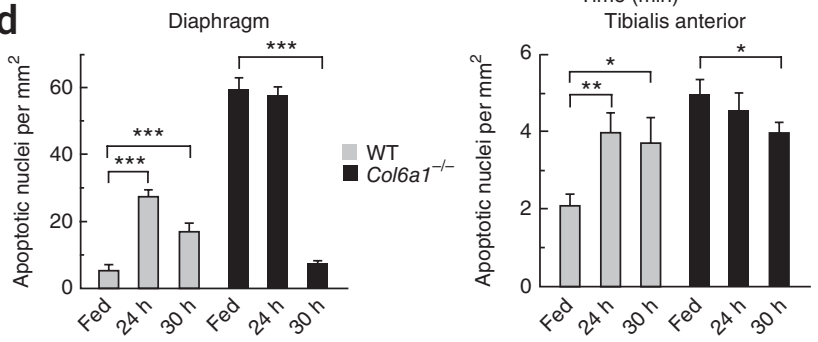

Next, we investigated the signaling pathways involved in autophagy regulation. In skeletal muscle, autophagy and Bnip3 expression are regulated by the Akt-forkhead box protein $\mathrm{O} 3$ axis, a pathway that also regulates the atrophy-related ubiquitin ligase atrogin-1 (ref. 22). Similarly to Bnip3, upregulation of atrogin-1 was impaired in fasted Col6a1 $1^{-1-}$ tibialis anterior, whereas induction of muscle RING-finger protein-1, another atrophy-related ubiquitin ligase that is under nuclear factor- $\kappa \mathrm{B}$ control, was unaffected (Supplementary Fig. 2i). These findings suggest there is abnormal Akt signaling in fasted Col6a1 $1^{-/-}$muscles, and, indeed, starvation induced dephosphorylation of Akt in wild-type but not Col6a1 $1^{-/-}$ tibialis anterior (Fig. $\mathbf{1 j}$ and Supplementary Fig. $\mathbf{2 j}$ ). In agreement with the abnormal Akt phosphorylation, the mammalian target of rapamycin pathway, which negatively regulates autophagy $y^{2,23}$, remained active in fasted Colba1 ${ }^{-/-}$tibialis anterior, as indicated by the persistent phosphorylation of eukaryotic translation initiation factor $4 \mathrm{E}$-binding protein-1 (4E-BP1), a downstream target of this signaling axis (Fig. 1j). Fasted Col6a1 $1^{-1-}$ diaphragm showed dephosphorylation of Akt and 4E-BP1, albeit to a lesser extent than starved wild-type muscles, and this explains why autophagy is partially activated in diaphragm after 24 -h fasting (Fig. $\mathbf{1 j}$ and Supplementary Fig. 2j).

Recent studies have shown that constitutive Akt expression in wild-type muscle inhibits autophagosome formation ${ }^{15}$. To get further evidence of the impact of abnormal Akt signaling, we investigated the phenotype of mice expressing a muscle-specific, inducible Akt transgene, resulting in chronic Akt activation. In agreement with our findings in Col6a1 ${ }^{-1-}$ mice, chronic Akt activation in otherwise
Figure 3 Induction of autophagy ameliorates the dystrophic phenotype. (a) Electron micrographs of diaphragm from Col6a1 $1^{-/-}$mice in fed conditions (left) and after $30 \mathrm{~h}$ starvation (right). Abnormal mitochondria (arrowheads) and dilated sarcoplasmic reticulum cisternae (arrows) in myofibers of the fed Col6a $1^{-1-}$ mice are indicated. Scale bar, $1 \mu \mathrm{m}$. (b) Percentage of myofibers with morphologically altered mitochondria in diaphragm of fed, 24-h-fasted and 30-h-fasted mice ${ }^{* * *} P<0.001$; $n=5$, each group). Error bars indicate s.d. (c) Mitochondrial response to oligomycin in myofibers isolated from FDB muscles of fed and fasted mice. Where indicated, $6 \mu \mathrm{M}$ oligomycin (arrow) or $4 \mu \mathrm{M}$ of the protonophore carbonylcyanide- $p$-trifluoromethoxyphenyl hydrazone (FCCP) (arrowhead) were added. Each trace represents the tetramethylrhodamine methyl ester (TMRM) fluorescence of a single fiber. The fraction of myofibers with depolarizing mitochondria is indicated for each condition, where fibers are considered as depolarizing when they lose more than $10 \%$ of initial value of TMRM fluorescence after oligomycin addition ( $n=5$, each group). (d) Quantification of TUNEL-positive nuclei in diaphragm (left) and tibialis anterior (right) of fed, 24-h-fasted and 30-h-fasted mice $\left({ }^{* *} P<0.001,{ }^{* *} P<0.01,{ }^{*} P<0.05 ; n=5\right.$, each group). Error bars indicate s.e.m.

wild-type mice led to decreased Bnip3 and beclin-1 protein amounts and resulted in a dystrophic phenotype characterized by p62 aggregates, vacuolated fibers and centrally located myonuclei (Supplementary Fig. 3). These findings support a role for the persistence of Akt activation in autophagy inhibition and in the development of the dystrophic phenotype in Col6a1 $1^{-/-}$mice.

Because 24-h fasting was not sufficient to trigger a robust Akt dephosphorylation and full activation of autophagy in Colba1 ${ }^{-1-}$ muscles, we investigated whether prolonged starvation could reactivate the autophagic process and confer some beneficial effects on the dystrophic phenotype. Starvation for $30 \mathrm{~h}$ triggered LC3 lipidation and increased beclin-1 and Bnip3 protein amounts in both diaphragm and tibialis anterior of Col6a1 $1^{-/-}$mice (Fig. 2a,b and Supplementary Fig. 4a,b). Bnip3 mRNA levels were also increased by 30 -h fasting, whereas Becn1 transcripts were unaffected (Fig. 2c and Supplementary Fig. 4c). Similarly, Vps34 was induced in Col6a1 $1^{-1-}$ muscles (Supplementary Fig. 4d,e). As with 24-h fasting, $\mathrm{Bcl}-\mathrm{X}_{\mathrm{L}}$ and Bax expression did not change between wildtype and Col6a1 $1^{-1-}$ muscles, whereas the Bcl-2 protein amount was increased in 30-h-fasted Col6a1 ${ }^{-1-}$ tibialis anterior (Supplementary Fig. 4f). Therefore, high expression of Bcl-2 did not prevent reactivation of autophagy in Col6a1 ${ }^{-/-}$mice. Prolonged starvation attenuated the differences between wild-type and Col6a ${ }^{-/-}$muscles in the phosphorylation of Akt (Fig. 2d). Atrogin-1 mRNA expression was induced in 30-h-fasted Col6a1 $1^{-1-}$ mice (Supplementary Fig. 4g). Detection of LC3-positive vesicles by fluorescence microscopy in YFP-LC3-transfected fibers and by immunoelectron microscopy confirmed that 30 -h fasting elicited substantial autophagosome formation in both wild-type and Col6a $1^{-1-}$ myofibers (Fig. 2e-g and Supplementary Fig. 4h).

Prolonged starvation resulted in the amelioration of myofiber abnormalities in Col6a1 $1^{-1-}$ mice (Fig. 3a). Indeed, Col6a1 $1^{-1-}$ diaphragms showed a significant rescue of ultrastructural alterations of mitochondria and sarcoplasmic reticulum (Fig. 3a,b and Supplementary Fig. $\mathbf{4 h - j}$ ). Moreover, the percentage of FDB myofibers showing mitochondrial depolarization was substantially lower, and the amount of TUNEL-positive-nuclei in Col6a1 ${ }^{-1-}$ muscles became close to that of control muscles (Fig. 3c,d). Wild-type mice showed some degree of muscle alterations after fasting (Fig. $\mathbf{3 b}$-d and Supplementary Fig. $\mathbf{4 h}, \mathbf{j}$ ), in keeping with the observation that excessive autophagy contributes to muscle wasting ${ }^{15,24}$. 
Figure 4 Beclin-1 protein abundance is decreased in muscle biopsies of subjects with UCMD or Bethlem myopathy, and its expression counteracts muscle apoptosis in Col6a1 $1^{-1-}$ mice. $(\mathbf{a}, \mathbf{b})$ Quantification of TUNELpositive nuclei in wild-type and Col6a ${ }^{-1-}$ tibialis anterior transfected with a vector expressing scramble or Becn1 shRNA (a) or Bnip3 shRNA (b). Western blot confirmation of RNAi-mediated knockdown of Becn1 and Bnip3 in tibialis anterior of wild-type mice is included above each set of graphs $\left({ }^{* *} P<0.001 ;{ }^{* *} P<0.01 ;{ }^{*} P<0.05 ; n=10\right.$, each group). Error bars indicate s.e.m. (c) Fluorescence microscopy of tibialis anterior cryosections from wild-type and Col6a1 ${ }^{-1-}$ mice transfected with beclin-1 and YFP-LC3 expression vectors and maintained in fed condition.

LC3 puncta (arrowheads) are indicated. Scale bar, $50 \mu \mathrm{m}$. (d) Right, quantification of TUNEL-positive nuclei in transfected and nontransfected myofibers of wild-type and Col6a1 $1^{-1-}$ tibialis anterior muscle after in vivo transfection with beclin-1-EGFP expression vector. Transfected fibers were revealed by fluorescence microscopy (left). Scale bar, $50 \mu \mathrm{m}$ (***P<0.001; $n=10$, each group). Error bars indicate s.e.m. (e) Western blot for beclin-1 and Bnip3 in protein lysates of human muscle biopsies from two healthy (normal) controls $(\mathrm{C} 1, \mathrm{C} 2)$, five individuals with UCMD (UCMD1-5) and four individuals with Bethlem myopathy (BM1-4). Data are representative of three independent experiments.

\section{Restoration of beclin-1 ameliorates muscle phenotype}

To further investigate the contribution of beclin-1 and Bnip3 in the pathogenesis of Col6 $\mathrm{al}^{-/-}$muscles, we performed in vivo loss-of-function experiments. Adult tibialis anterior was transfected with expression plasmids encoding shRNAs targeting Becn 1 and Bnip 3 transcripts ${ }^{24}$. $B e c n 1$ knockdown for 2 weeks led to a marked increase in apoptotic nuclei in myofibers of wild-type mice and completely prevented the significant decrease of TUNEL-positive nuclei observed in 30-h-fasted Col6a1 $1^{-/-}$mice (Fig. 4a). Conversely, Bnip3 knockdown for 2 weeks did not substantially impinge on the incidence of TUNEL-positive nuclei of wild-type mice and had a minor effect on 30-h-starved Col6a1 ${ }^{-1-}$ mice when compared to Becn 1 knockdown (Fig. 4b). Together with the above findings showing that autophagy flux is impaired in Col6a1 $1^{-/-}$muscles, these data indicate that the inefficient autophagy and subsequent muscle apoptosis observed in these mice is due to beclin-1 inhibition.

To support the pathogenetic role of beclin-1 deficiency in myofiber degeneration, we carried out in vivo transfection of tibialis anterior with an expression construct coding for beclin-1. Transfection of the beclin-1 construct was able to activate autophagy in both wild-type and Col6a1-1- muscles, as revealed by concurrent transfection of YFP-LC3 and formation of LC3-positive puncta (Fig. 4c). Moreover, beclin-1 overexpression led to a marked decrease of TUNEL-positive nuclei in myofibers of Col6a1 $1^{-/-}$mice, whereas it did not affect wildtype muscles (Fig. 4d). Therefore, correction of beclin-1 levels in Col6a $1^{-/-}$muscle fibers is sufficient to reactivate autophagy and thus prevent apoptotic degeneration.

To assess whether the findings obtained in the Col6a1-null mouse model also apply to human collagen VI disorders, we investigated muscle biopsies derived from five subjects with UCMD and four subjects with Bethlem myopathy (Supplementary Table 1). The amounts of both beclin-1 and Bnip3 proteins were decreased in subjects with UCMD and Bethlem myopathy when compared to healthy (normal) controls. Individuals with UCMD had very low beclin-1 levels, whereas individuals with Bethlem myopathy, whose dystrophic phenotype is milder, showed a less prominent decrease in the amount of beclin-1, which seemed closer but not identical to amounts in controls. Bnip3 protein abundance was also lower in samples from UCMD and Bethlem myopathy individuals compared to those in controls, albeit the reductions are much less prominent than that seen for beclin-1 abundance between disease samples and controls (Fig. 4e and Supplementary Fig. 5a). a
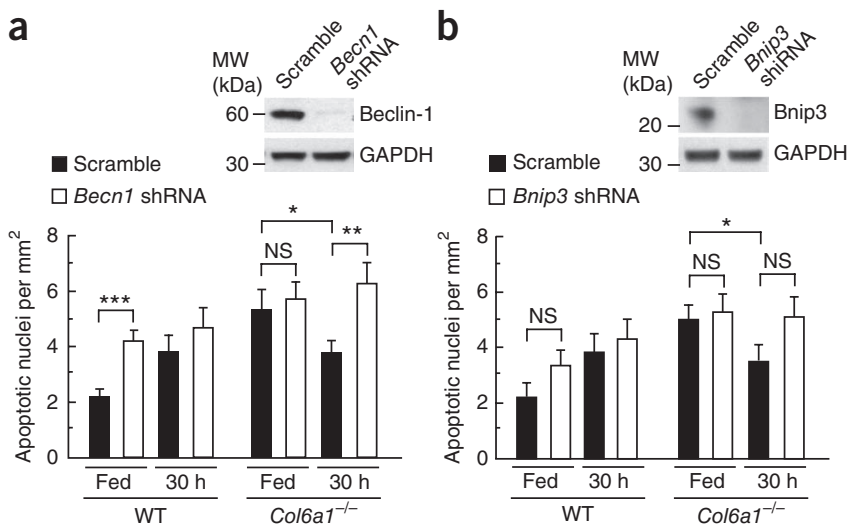

$\square$ Bnip3 shRNA

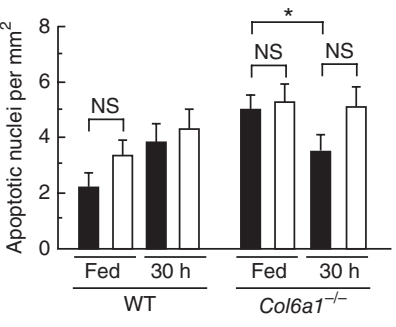

C

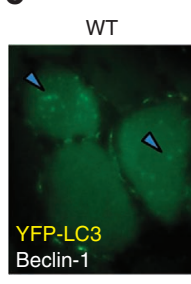

d

Nontransfected fibers

\section{e $\quad M$}

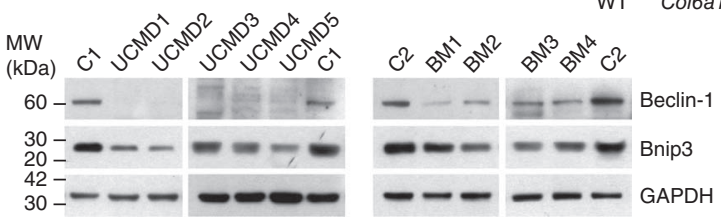
$\square$ Transfected fibers

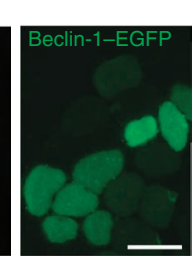
$\square$ Transfected fibers

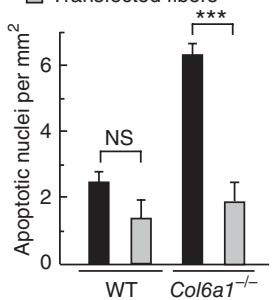

\section{Autophagy reactivation ameliorates muscle pathology}

Given that prolonged starvation reactivated autophagy and blocked apoptotic degeneration in Col6a1 $1^{-/-}$muscles, we investigated whether milder and long-lasting dietary regimens were able to activate autophagy and ameliorate muscle morphology and function. Depletion of amino acids strongly induces autophagy ${ }^{25-27}$. Conversely, supplementation of protein-free diets with amino acids suppresses muscle protein degradation through inhibition of autophagy $y^{28}$. To allow for a long-term response, we fed mice with a specifically designed lowprotein diet (LPD) (Supplementary Table 2). Four weeks of LPD was able to ameliorate the dystrophic features of Col6 $\mathrm{a}^{-/-}$mice (Fig. 5a,b and Supplementary Fig. 6). LPD induced autophagy and did not have any significant effect on the ubiquitin-proteasome system as shown by atrogin-1 expression (Supplementary Fig. 6c). Diaphragm and tibialis anterior of both wild-type and Col6a1 $1^{-/-}$LPD-fed mice showed LC3 lipidation and increased beclin-1 and Bnip3 protein amounts, consistent with the formation of autophagosomes (Fig. 5c,d and Supplementary Fig. 6a,b). Activation of autophagy by LPD led to removal of structurally abnormal organelles (Fig. 5b and Supplementary Fig. 6e). Similarly to prolonged starvation, induction of autophagy by LPD led to a marked recovery of the dystrophic alterations of Col6a1 $1^{-1-}$ mice and produced some muscle alterations in wild-type mice (Fig. 5e,f and Supplementary Fig. 6e-g). The percentages of myofibers showing mitochondrial depolarization and TUNEL positivity were significantly lower in LPD-fed Col6a1 $1^{-/-}$mice compared to Col6a1 $1^{-1-}$ mice fed with normal diet and were similar to (or lower than) those observed in LPDfed wild-type mice (Fig. 5f and Supplementary Fig. 6g). Autophagy reactivation also ameliorated the histological features of Col6a1 $1^{-/-}$ muscles, resulting in a more uniform myofiber size (Supplementary Fig. 6h). Moreover, long-term induction of autophagy by LPD also 

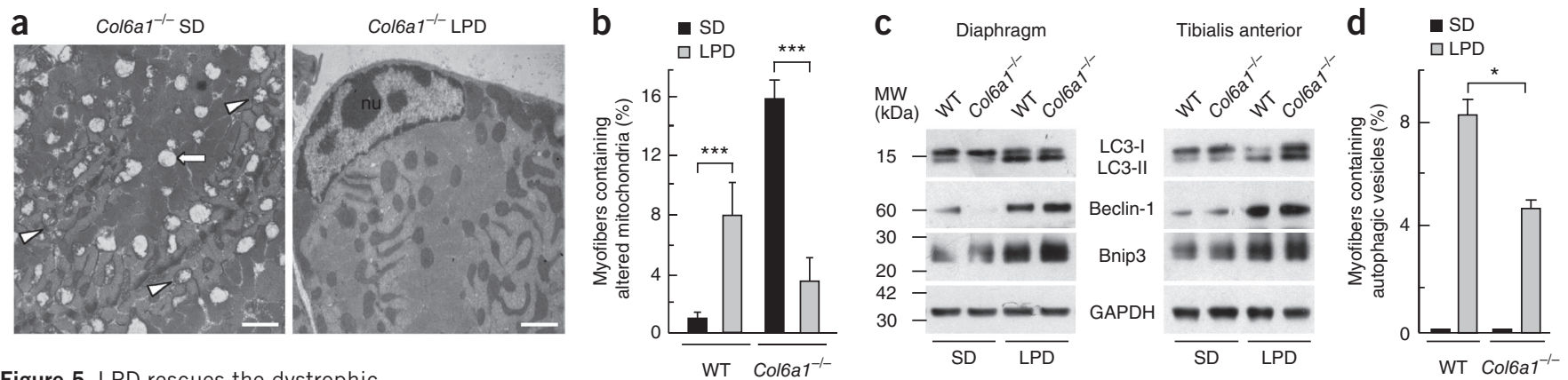

Figure 5 LPD rescues the dystrophic phenotype of Col6a1--I- mice. (a) Electron micrographs of diaphragm from standard diet (SD)-fed and LPD-fed Col6a1 ${ }^{-1-}$ mice. Abnormal mitochondria (arrowheads) and dilated sarcoplasmic reticulum cisternae (arrows) in Col6a1 ${ }^{-1-}$ myofibers are indicated. nu, nucleus. Scale bar, $500 \mathrm{~nm}$. (b) Percentage of myofibers with morphologically altered mitochondria in diaphragms of mice fed with SD or LPD. (*** $P<0.001 ; n=5$, each group). Error bars indicate s.d. (c) Western blot for LC3, beclin-1 and Bnip3 in diaphragm (left) and tibialis anterior (right) of mice fed with SD or LPD. (d) Percentage of myofibers containing autophagic vesicles in diaphragm of wild-type and Col6a1 $1^{-/}$ mice fed with SD or LPD ( ${ }^{*} P<0.05 ; n=5$

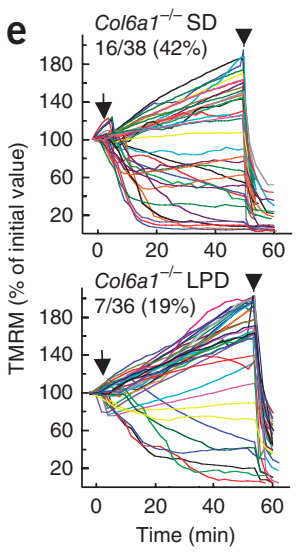

f

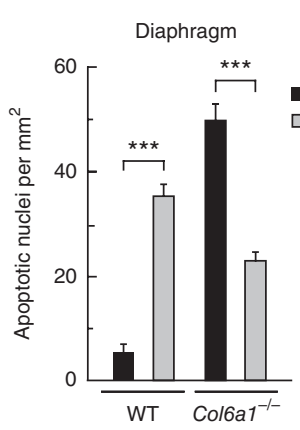

g

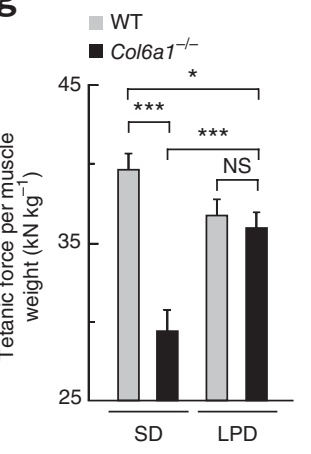
each group). Error bars indicate s.d. (e) Mitochondrial response to oligomycin in FDB myofibers of Col6a1 ${ }^{-1-}$ fed with SD or LPD. The fraction of depolarizing myofibers is provided as in Figure $3 \mathbf{c}(n=5$, each group). (f) Quantification of TUNEL-positive nuclei in diaphragm (left) and tibialis anterior (right) of mice fed with SD or LPD (*** $P<0.001 ;{ }^{* *} P<0.01 ;{ }^{*} P<0.05 ; n=5$, each group). Error bars indicate s.e.m. (g) In vivo tetanic force measurements in the gastrocnemius muscle of mice fed with SD or LPD (*** $P<0.001 ;{ }^{*} P<0.05 ; n=12$, each group). Error bars indicate s.e.m.

improved muscle strength of Col6a1 $1^{-/-}$mice, as indicated by the significant increase of specific force measured in gastrocnemius muscle (Fig. 5g and Supplementary Fig. 6i). Thus, reactivation of autophagy by dietary approaches is sufficient to improve both morphology and function of Col6a1 $1^{-/-}$dystrophic muscles.

\section{Drugs inducing autophagy rescue the dystrophic phenotype}

As reactivation of autophagy by dietary restriction showed a therapeutic potential in Col6a1 $1^{-/-}$mice, we further explored this possibility with pharmacological tools. Rapamycin is a well-known inducer of autophagy that can be used for in vivo treatments ${ }^{17}$. Treatment of Col6a1 $1^{-1-}$ mice with rapamycin for $15 \mathrm{~d}$ decreased myofiber degeneration and removed abnormal organelles (Fig. 6a-c and Supplementary Fig. 7a). Cyclosporin A has been reported to modulate autophagy $y^{7,29,30}$, and we previously demonstrated that this drug rescues the dystrophic phenotype of Col6a1-1- mice by desensitizing the mitochondrial permeability transition pore ${ }^{9}$. Because reactivation of autophagy in $\mathrm{Col}_{6} \mathrm{a1}^{-/-}$mice led to a marked amelioration of the phenotype, we investigated whether treatment with cyclosporin A would have any effect on autophagy. Notably, cyclosporin A induced autophagy in Col6a1 $1^{-1-}$ muscles, with a concomitant block of apoptotic degeneration and recovery of muscle strength (Fig. 6d-g and Supplementary Fig. 7b-d). Moreover, cylosporin A treatment restored beclin-1 and Bnip3 induction, LC3 lipidation and Akt dephosphorylation in 24-h-fasted Col6a1 $1^{-/-}$ muscles (Fig. 6d and Supplementary Fig. 7e). These data suggest that the robust beneficial effect of cyclosporin A in collagen VIdeficient mice is linked to both restoration of mitochondrial function $^{9}$ and autophagy induction.

\section{DISCUSSION}

Previous studies have shown that the muscle pathology of collagen VInull mice and humans with UCMD or Bethlem myopathy is strictly related to ultrastructural alterations of organelles, mitochondrial dysfunction and spontaneous apoptosis in skeletal muscles ${ }^{9,10}$. Here we show that autophagy is impaired in Col6a1 ${ }^{-/-}$muscles and that the failure of this process has a major role in the pathogenesis of the dystrophic phenotype, causing accumulation of abnormal organelles and apoptotic degeneration of muscle fibers. Forced induction of autophagy is able to reactivate the process, achieving a beneficial response in Col6a1 $1^{-1-}$ mice. Thus, prompt elimination of defective organelles is essential to protect Col6a1 $1^{-1-}$ myofibers from the harmful consequences of dysfunctional mitochondria and excessive apoptosis (Supplementary Fig. 8a). Indeed, accumulation of abnormal mitochondria in Col6a1 $1^{-1-}$ muscles is associated with oxidative stress and increased production of reactive oxygen species, which contribute to trigger apoptotic response ${ }^{31}$. Our findings indicate that a proper autophagic flux is crucial for the clearance of damaged organelles and the maintenance of muscle homeostasis. Moreover, we also point out that both defective and excessive autophagy lead to muscle degeneration, in keeping with the intricate interplay between autophagic and apoptotic pathways shown in different systems ${ }^{1-3,32}$ (Supplementary Fig. 8b). In agreement with our model, musclespecific inactivation of autophagy genes results in muscle atrophy with abnormal organelles ${ }^{33,34}$.

In certain storage diseases and vacuolar myopathies, a reduced 'offrate' of autophagy results from mutations of genes coding for proteins involved in lysosomal function, leading to accumulation of autophagic vesicles $^{1,3,35,36}$. Conversely, our findings suggest that the impaired 
Figure 6 Pharmacological treatments induce autophagy and ameliorate the myopathic phenotype of Col6a $1^{-1-}$ mice. (a) Quantification of TUNEL-positive nuclei in diaphragm (left) and tibialis anterior (right) of wild-type and Col6a1 $1^{-1-}$ mice treated with vehicle (ethanol) or with rapamycin (Rap) for $15 \mathrm{~d}\left({ }^{* *} P<0.001 ;{ }^{*} P<0.05 ; n=5\right.$, each group). Error bars indicate s.e.m.

(b) Representative micrographs of a transverse section of diaphragm from untreated (left) and rapamycin-treated (right) Col6a $1^{-/-}$mice. Abnormal mitochondria (white arrowheads) and dilated sarcoplasmic reticulum (white arrows) in the muscle of untreated mice are indicated, as is autophagosome formation in the treated mice (inset). Scale bar, $500 \mathrm{~nm}$. (c) Percentage of myofibers with morphologically altered mitochondria in the diaphragm of Col6a1-l- mice treated with rapamycin (+) or left untreated $(-)$ for $15 \mathrm{~d}(* * * P<0.001$; $n=5$ each group). Error bars indicate s.d.

(d) Western blot analysis for LC3, beclin-1 and Bnip3 in diaphragm (left) and tibialis anterior (right) of wild-type and Col6a1 $1^{-1-}$ mice treated with vehicle (olive oil) (-) or with cyclosporin $A$ $(\mathrm{CsA})(+)$ for $4 \mathrm{~d}$ and maintained in fed or 24-h-fasting conditions. (e) Quantification of TUNEL-positive nuclei in diaphragm (left) and tibialis anterior (right) of wild-type and Col6a1 $1^{-l-}$ mice treated with vehicle or with cyclosporin A for $4 \mathrm{~d}(* * * P<0.001$;

$n=4$, each group). Error bars indicate s.e.m. (f) In vivo tetanic force measurements in the gastrocnemius muscle of mice treated with vehicle or with cyclosporin $A$ for $10 \mathrm{~d}$ $\left({ }^{* *} P<0.001 ;{ }^{* *} P<0.01 ;{ }^{*} P<0.05 ; n=8\right.$, each group). Error bars indicate s.e.m.
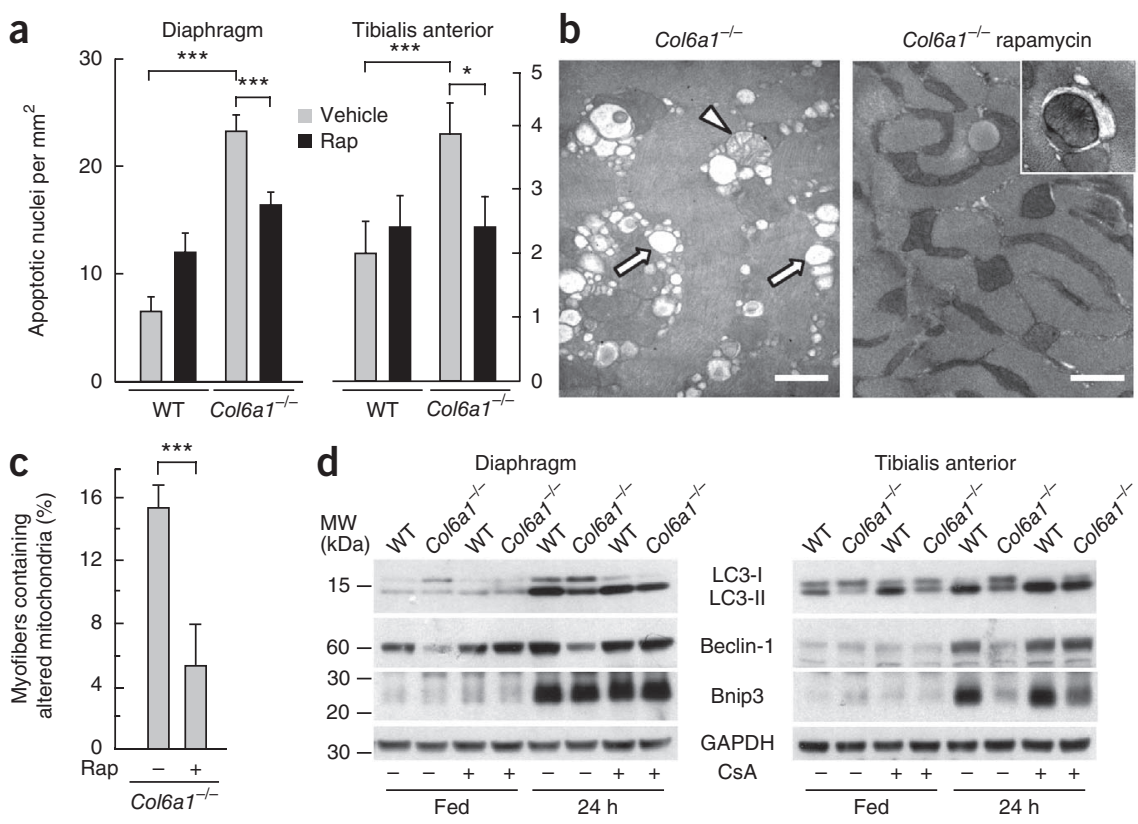

e

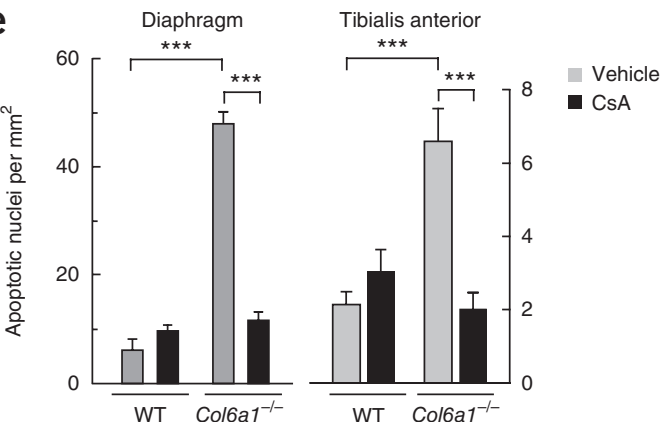

f

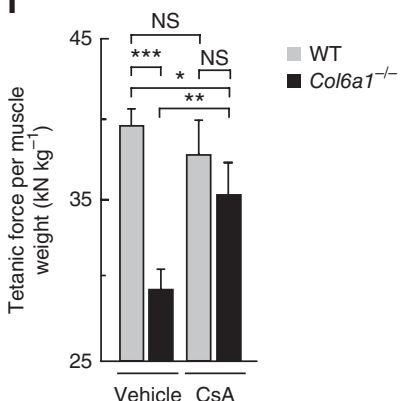

autophagic flux of collagen VI dystrophic muscles results from a decreased 'on-rate', with defective formation of autophagosomes. Indeed, we have found that amounts of two major autophagic effector proteins, beclin-1 and Bnip3, are reduced in Col6a1 $1^{-1-}$ muscles. Although Bnip3 induction is sufficient to trigger autophagy ${ }^{15}$, our data suggest that the concomitant beclin-1 upregulation is required for a proper autophagy induction. Thus, beclin-1 seems to be necessary and sufficient for basal autophagy and for its correct induction, whereas Bnip3 is able to trigger autophagy in the presence of appropriate protein levels of beclin-1.

The demonstration that muscles of Col6a1 $1^{-/-}$mice are characterized by inefficient autophagy has a major impact on the understanding of the pathogenesis of collagen VI muscular dystrophies. Muscle biopsies of subjects with UCMD and Bethlem myopathy show reduced protein amounts of beclin-1 and Bnip3. Notably, forced reactivation of autophagy in the mouse model by nutritional and pharmacological approaches is able to rescue the morphological and functional aspects of the dystrophic phenotype. This opens new possibilities for targeted therapeutic approaches aimed at combating muscle loss in UCMD and Bethlem myopathy that add to desensitization of the mitochondrial permeability transition pore through cyclophilin D inhibition ${ }^{10,37,38}$.

In more general terms, our results support a view that points at the activators of autophagic process and at clearance systems as new therapeutic targets for avoiding accumulation of toxic molecules and damaged organelles in skeletal muscle. Moreover, the finding that modulation of autophagy through various nutritional and pharmacological treatments is beneficial for collagen VI dystrophic muscles paves the way for investigating autophagy defects in other muscular dystrophies.

\section{METHODS}

Methods and any associated references are available in the online version of the paper at http://www.nature.com/naturemedicine/.

Note: Supplementary information is available on the Nature Medicine website.

\section{ACKNOWLEDGMENTS}

We thank N. Bergamin for her involvement in the initial study, P. Braghetta for help with mouse manipulations, E. Rizzo and S. Castagnaro for histology, S. Cogliati for assistance with mitochondria isolation and F. Gualandi for muscle biopsies. We are grateful to N. Heintz (Rockefeller University) for supplying the beclin-1-EGFP expression construct and E. Kominami (Juntendo University School of Medicine) for the YFP-LC3 construct. This work was supported by the Telethon Foundation (GGP08107 and TCP04009), the Italian Ministry of University and Research, Association Francaise contre les Myopathies and the EU (BIO-NMD and MYOAGE).

\section{AUTHOR CONTRIBUTIONS}

P.G. performed biochemical analyses, autophagy assays, mouse treatments, analysis and interpretation of data, and contributed to manuscript preparation. L.C. carried out RNA analysis, muscle transfections, molecular biology, analysis and interpretation of data, and contributed to manuscript preparation. P.S. performed electron microscopy. M.C. performed TUNEL and histology. A.A. performed tetramethylrhodamine methyl ester (TMRM) analysis. E.B. carried out muscle transfections and mitochondria isolation. B.B. analyzed muscle mechanics. A.U. performed TUNEL analysis. T.T. genotyped and maintained mice. L.M., 
P. Bernardi and N.M.M. were involved in data analysis. P. Bonaldo and M.S. designed the study, analyzed data and wrote the paper. All authors discussed the results and commented on the manuscript.

\section{COMPETING FINANCIAL INTERESTS}

The authors declare no competing financial interests.

Published online at http://www.nature.com/naturemedicine/.

Reprints and permissions information is available online at http://npg.nature.com/ reprintsandpermissions/.

1. Levine, B. \& Kroemer, G. Autophagy in the pathogenesis of disease. Cell 132, 27-42 (2008).

2. Maiuri, M.C., Zalckvar, E., Kimchi, A. \& Kroemer, G. Self-eating and self-killing: crosstalk between autophagy and apoptosis. Nat. Rev. Mol. Cell Biol. 8, 741-752 (2007).

3. Mizushima, N., Levine, B., Cuervo, A.M. \& Klionsky, D.J. Autophagy fights disease through cellular self-digestion. Nature 451, 1069-1075 (2008).

4. Nakai, A. et al. The role of autophagy in cardiomyocytes in the basal state and in response to hemodynamic stress. Nat. Med. 13, 619-624 (2007).

5. Green, D.R. \& Kroemer, G. The pathophysiology of mitochondrial cell death. Science 305, 626-629 (2004).

6. Bernardi, P. et al. The mitochondrial permeability transition from in vitro artifact to disease target. FEBS J. 273, 2077-2099 (2006).

7. Kim, I., Rodriguez-Enriquez, S. \& Lemasters, J.J. Selective degradation of mitochondria by mitophagy. Arch. Biochem. Biophys. 462, 245-253 (2007).

8. Lampe, A.K. \& Bushby, K.M. Collagen VI related muscle disorders. J. Med. Genet. 42, 673-685 (2005)

9. Irwin, W.A. et al. Mitochondrial dysfunction and apoptosis in myopathic mice with collagen VI deficiency. Nat. Genet. 35, 367-371 (2003).

10. Angelin, A. et al. Mitochondrial dysfunction in the pathogenesis of Ullrich congenital muscular dystrophy and prospective therapy with cyclosporins. Proc. Natl. Acad. Sci. USA 104, 991-996 (2007).

11. Long, Y.C. \& Zierath, J.R. AMP-activated protein kinase signaling in metabolic regulation. J. Clin. Invest. 116, 1776-1783 (2006).

12. Mizushima, N., Yamamoto, A., Matsui, M., Yoshimori, T. \& Ohsumi, Y. In vivo analysis of autophagy in response to nutrient starvation using transgenic mice expressing a fluorescent autophagosome marker. Mol. Biol. Cell 15, 1101-1111 (2004).

13. Mizushima, N., Yoshimori, T. \& Levine, B. Methods in mammalian autophagy research. Cell 140, 313-326 (2010).

14. Klionsky, D.J. et al. Guidelines for the use and interpretation of assays for monitoring autophagy in higher eukaryotes. Autophagy 4, 151-175 (2008).

15. Mammucari, C. et al. FoxO3 controls autophagy in skeletal muscle in vivo. Cell Metab. 6, 458-471 (2007)

16. Schmalbruch, $H$. The early changes in experimental myopathy induced by chloroquine and chlorphentermine. J. Neuropathol. Exp. Neurol. 39, 65-81 (1980).

17. Rubinsztein, D.C., Gestwicki, J.E., Murphy, L.O. \& Klionsky, D.J. Potential therapeutic applications of autophagy. Nat. Rev. Drug Discov. 6, 304-312 (2007).
18. Bjørkøy, G. et al. p62/SQSTM1 forms protein aggregates degraded by autophagy and has a protective effect on huntingtin-induced cell death. J. Cell Biol. 171, 603-614 (2005).

19. Hamacher-Brady, A. et al. Response to myocardial ischemia/reperfusion injury involves Bnip3 and autophagy. Cell Death Differ. 14, 146-157 (2007).

20. Sandoval, H. et al. Essential role for Nix in autophagic maturation of erythroid cells. Nature 454, 232-235 (2008).

21. Pattingre, S. et al. Bcl-2 antiapoptotic proteins inhibit beclin-1-dependent autophagy. Cell 122, 927-939 (2005).

22. Sandri, M. et al. FoxO transcription factors induce the atrophy-related ubiquitin ligase atrogin-1 and cause skeletal muscle atrophy. Cell 117, 399-412 (2004)

23. Shintani, T. \& Klionsky, D.J. Autophagy in health and disease: a double-edged sword. Science 306, 990-995 (2004).

24. Romanello, V. et al. Mitochondrial fission and remodelling contributes to muscle atrophy. EMBO J. 29, 1774-1785 (2010).

25. Codogno, P. \& Meijer, A.J. Autophagy and signaling: their role in cell survival and cell death. Cell Death Differ. 12 Suppl 2, 1509-1518 (2005).

26. Mizushima, N. Autophagy: process and function. Genes Dev. 21, 2861-2873 (2007).

27. Mortimore, G.E. \& Poso, A.R. Intracellular protein catabolism and its control during nutrient deprivation and supply. Annu. Rev. Nutr. 7, 539-564 (1987).

28. Sugawara, T., Ito, Y., Nishizawa, N. \& Nagasawa, T. Regulation of muscle protein degradation, not synthesis, by dietary leucine in rats fed a protein-deficient diet. Amino Acids 37, 609-616 (2009).

29. Yoo, Y.M. \& Jeung, E.B. Melatonin suppresses cyclosporine A-induced autophagy in rat pituitary GH3 cells. J. Pineal Res. 48, 204-211 (2010).

30. Pallet, N. et al. Autophagy protects renal tubular cells against cyclosporine toxicity. Autophagy 4, 783-791 (2008).

31. Menazza, S. et al. Oxidative stress by monoamine oxidases is causally involved in myofiber damage in muscular dystrophy. Hum. Mol. Genet. published online, doi:10.1093/hmg/ddq339 (17 August 2010).

32. Boya, P. et al. Inhibition of macroautophagy triggers apoptosis. Mol. Cell. Biol. 25 , 1025-1040 (2005).

33. Masiero, E. et al. Autophagy is required to maintain muscle mass. Cell Metab. 10 , 507-515 (2009).

34. Wu, J.J. et al. Mitochondrial dysfunction and oxidative stress mediate the physiological impairment induced by the disruption of autophagy. Aging 1, 425-437 (2009).

35. Ramachandran, N. et al. VMA21 deficiency causes an autophagic myopathy by compromising V-ATPase activity and Iysosomal acidification. Cell 137, 235-246 (2009).

36. Malicdan, M.C., Noguchi, S., Nonaka, I., Saftig, P. \& Nishino, I. Lysosomal myopathies: an excessive build-up in autophagosomes is too much to handle. Neuromuscul. Disord. 18, 521-529 (2008).

37. Palma, E. et al. Genetic ablation of cyclophilin D rescues mitochondrial defects and prevents muscle apoptosis in collagen VI myopathic mice. Hum. Mol. Genet. 18, 2024-2031 (2009).

38. Merlini, L. et al. Cyclosporin A corrects mitochondrial dysfunction and muscle apoptosis in patients with collagen VI myopathies. Proc. Natl. Acad. Sci. USA 105 5225-5229 (2008). 


\section{ONLINE METHODS}

Mice. We backcrossed Col6a1 ${ }^{+/-}$mice in the inbred C57BL/6J strain (Charles River) for eight generations ${ }^{9}$. We performed all experiments in 16- to 24 -weekold mice and compared age-matched Col6a1 ${ }^{-/-}$(collagen VI-null) and Col6a1 ${ }^{+/+}$ (wild-type) mice. We housed mice in individual cages in an environmentally controlled room $\left(23^{\circ} \mathrm{C}, 12\right.$-h light-dark cycle) and provided food and water ad libitum. For starvation experiments, we removed chow in the morning and maintained mice for 6-30 $\mathrm{h}$ with no food but free access to water. We fed mice with either SD (Laboratorio Dottori Piccioni) or LPD (TestDiet) (Supplementary Table 2). Muscle-specific inducible Akt-ER-Cre transgenic mice were previously described ${ }^{15}$, and we achieved transgene activation by tamoxifen administration in the chow (Harlan). Mouse procedures were approved by the Ethics Committee of the University of Padova and authorized by the Italian Ministry of Health.

Drug treatments. We subjected wild-type and Col6a1 ${ }^{-1-}$ mice to intraperitoneal injection with either chloroquine diphosphate ( $50 \mathrm{mg}$ per kg body weight; Sigma) every $24 \mathrm{~h}$ for $10 \mathrm{~d}$; cyclosporine A (5 mg per kg body weight; Novartis) every $12 \mathrm{~h}$ for $4 \mathrm{~d}$ or $10 \mathrm{~d}$ or rapamycin ( $2 \mathrm{mg}$ per kg body weight; LC Laboratories) every $24 \mathrm{~h}$ for $15 \mathrm{~d}$.

Muscle in vivo transfection. We performed in vivo transfection experiments by intramuscular injection of expression plasmids in tibialis anterior followed by electroporation as previously described ${ }^{22}$. We used the following expression constructs: YFP-LC3 (ref. 39), beclin-1-EGFP ${ }^{40}$ and beclin-1. For the preparation of the beclin-1 expression construct, we amplified Becn 1 cDNA by PCR from the beclin-1-EGFP plasmid using primers 5'-CTATGGAGGGGTCTAAGGC-3' (forward) and $5^{\prime}$-TCACTTGTTATAGAACTGTGAGG-3' (reverse) and cloned the amplified sequence into the HindIII and XbaI sites of the pcDNA3.1 (Invitrogen) expression vector. We carried out RNAi-mediated knockdown by transfection of shRNA constructs targeting Map1lc3b (ref. 15), Bnip3 (ref. 15) and Becn1. For Becn1, we used a commercial kit containing oligonucleotides against Becn 1 target sequences and the BLOCK-IT Pol II miR RNAi expression vector (Invitrogen).

Gene expression analyses. We prepared total RNA from skeletal muscle with the Promega SV Total RNA Isolation kit. We generated cDNA products with SuperScript III reverse transcriptase (Invitrogen) and analyzed them by qRTPCR with the QuantiTect SYBR Green PCR kit (Qiagen). We normalized all data to Gapdh expression. Oligonucleotide primers used for qRT-PCR are listed in Supplementary Table 3.

Fluorescence microscopy and transmission electron microscopy. We fixed muscle cryosections with ice-cold $4 \%$ paraformaldehyde, mounted the samples with Fluorescence Mounting Medium (DAKO) and examined them on a Leica DM5000B fluorescence microscope. For electron microscopy, we fixed and stained stretched diaphragms as previously described ${ }^{9}$. For statistical analysis, we studied at least 1,000 muscle fibers, obtained from two levels of three different tissue blocks for each diaphragm. We considered positive fibers presenting at least one mitochondrion with abnormal cristae or a portion of dilated sarcoplasmic reticulum. We performed immunoelectron microscopy according to previously published protocols ${ }^{41}$. We etched ultrathin sections with $3 \%$ sodium alcholate, treated them with $10 \% \mathrm{H}_{2} \mathrm{O}_{2}$ and incubated them overnight with LC3-specific antibody (LB 100-2220, Novus Biologicals). We visualized the antibody binding by incubation with 15 -nm colloidal gold-conjugated secondary antibody (Amersham).

Western blotting. We pulverized mouse frozen muscles and human muscle biopsies by grinding in liquid nitrogen, and we lysed and immunoblotted the samples as previously described ${ }^{22}$. When needed, we stripped and reprobed membranes. We used antibodies from Cell Signaling Technologies specific for the following proteins: 4E-BP1 (9452), phospho-4E-BP1 (Thr37 and Thr46) (2855), AMPK (2532), phospho-AMPK (Thr172) (2531), Akt (9272), phospho-Akt (Ser473) (4058), Bcl-X $\mathrm{X}_{\mathrm{L}}$ (2764), beclin-1 (3738), caspase-3 (9665), caspase-9 (9504), LC3 (2775), S6 (2212), phospho-S6 (Ser240 and Ser244) (2215). Antibodies to Bnip3 (B7931) and Vps34 (V9764) were from Sigma. Antibodies to Bax (sc-493) and TOM20 (sc-11415) were from Santa Cruz. Antibodies to Bcl-2 (610539) and calnexin (610523) were from BD Transduction Laboratories. Antibody to p62 (GP62-C) was from Progen. Antibody to GAPDH (MAB374) was from Chemicon International. We performed western blots in at least three independent experiments. We carried out densitometric quantification by the ImageJ software (US National Institutes of Health).

Isolation of skeletal myofibers and measure of mitochondrial membrane potential. We isolated muscle fibers from FDB muscle, and we measured mitochondrial membrane potential by epifluorescence microscopy on the basis of the accumulation of TMRM fluorescence, as previously described ${ }^{9,37}$. We considered fibers as depolarizing when they lost more than $10 \%$ of the initial value of TMRM fluorescence. We performed imaging with a Zeiss Axiovert 100 TV inverted microscope equipped with a 12-bit digital cooled charge-coupled device camera (Micromax, Princeton Instruments). We analyzed the data with MetaFluor imaging software (Universal Imaging).

Terminal deoxynucleotidyl transferase dUTP nick end labeling. We prepared sections ( $7-\mu \mathrm{m}$ thick) from diaphragm, after fixation with $4 \%$ paraformaldehyde and paraffin embedding, and from tibialis anterior muscles frozen in isopentane. We performed TUNEL assays with the ApopTag peroxidase in situ apoptosis detection system (Chemicon) ${ }^{9,37}$. For transfected muscles, we determined the number of TUNEL-positive nuclei in randomly selected fields by considering, separately, transfected and nontransfected fibers.

Muscle mechanics. We carried out in vivo determination of force and contraction kinetics of gastrocnemius muscle as previously described ${ }^{42}$.

Human samples. We froze muscle biopsies of children and adults in isopentane. Details on subjects included in the study are provided in Supplementary Table 1. All subjects provided informed consent and were previously diagnosed with UCMD and Bethlem myopathy according to the criteria of the European NeuroMuscular Center ${ }^{43}$ and by genetic analysis, which showed mutations in any of the COL6A1, COL6A2 and COL6A3 genes.

Statistical analyses. We expressed data as means \pm s.e.m. or as means \pm s.d. We determined statistical significance by unequal variance Student's $t$ test (for TUNEL assay), equal variance Student's $t$ test (for qRT-PCR and muscle mechanics) and Mann-Whitney test (for electron microscopy). A $P$ value of less than 0.05 was considered statistically significant.

39. Tanida, I. et al. HsAtg4B/HsApg4B/autophagin-1 cleaves the carboxyl termini of three human Atg8 homologues and delipidates microtubule-associated protein light chain 3- and GABAA receptor-associated protein-phospholipid conjugates. J. Biol. Chem. 279, 36268-36276 (2004).

40. Zhong, Y. et al. Distinct regulation of autophagic activity by Atg14L and Rubicon associated with beclin-1-phosphatidylinositol-3-kinase complex. Nat. Cell Biol. 11, 468-476 (2009).

41. Degrassi, A. et al. Transfer of HIV-1 to human tonsillar stromal cells following cocultivation with infected lymphocytes. AIDS Res. Hum. Retroviruses 10, 675-682 (1994).

42. Blaauw, B. et al. Akt activation prevents the force drop induced by eccentric contractions in dystrophin-deficient skeletal muscle. Hum. Mol. Genet. 17, 3686-3696 (2008).

43. Pepe, G. et al. Bethlem myopathy (BETHLEM) and Ullrich scleroatonic muscular dystrophy: 100th ENMC international workshop, 23-24 November 2001, Naarden, The Netherlands. Neuromuscul. Disord. 12, 984-993 (2002). 


\section{CORRIGENDA}

\section{Corrigendum: Autophagy is defective in collagen VI muscular dystrophies, and its reactivation rescues myofiber degeneration}

Paolo Grumati, Luisa Coletto, Patrizia Sabatelli, Matilde Cescon, Alessia Angelin, Enrico Bertaggia, Bert Blaauw, Anna Urciuolo, Tania Tiepolo, Luciano Merlini, Nadir M Maraldi, Paolo Bernardi, Marco Sandri \& Paolo Bonaldo

Nat. Med. 16, 1313-1320 (2010); published online 31 October 2010; corrected after print 2 March 2011

In the version of this article initially published, the affiliation of two of the authors was incorrectly listed. The Institute of Medical Genetics-National Research Council, Bologna, Italy should have been listed as the Institute of Molecular Genetics, National Research Council, c/o Rizzoli Orthopaedic Institute, Bologna, Italy. The error has been corrected in the HTML and PDF versions of the article. 